\title{
Study of Millimetre Wave Extended Interaction Oscillation using Pseudospark-sourced E-Beams
}

\author{
A.W. Cross ${ }^{1}$, H. Yin ${ }^{1}$, L. Zhang ${ }^{1}$, W. He ${ }^{1}$, G. Shu ${ }^{1}$, J. Zhao ${ }^{2}$, Y. Yin ${ }^{3}$, K. Ronald ${ }^{1}$ and A.D.R. Phelps ${ }^{1}$ \\ ${ }^{1}$ Department of Physics, SUPA, University of Strathclyde, Glasgow G4 0NG, Scotland, UK \\ ${ }^{2}$ High Voltage Division, School of Electrical Engineering, Xi'an Jiaotong University, Xi'an, 710049, China \\ ${ }^{3}$ School of Physical Electronics, University of Electronic Science \& Technology of China, Chengdu, China \\ 1a.w.cross@strath.ac.uk
}

\begin{abstract}
This article presents the investigation of millimetre wave extended interaction oscillation (EIO) using both pencil and sheet-shaped pseudospark (PS)-sourced electron beams. A Wband (75-110 GHz) pencil beam Extended Interaction Oscillator (EIO) was designed and constructed to operate for the first experiment with the output power of $38 \mathrm{~W}$ achieved from a fourgap PS discharge operating at $30.5 \mathrm{kV}$. To increase the output power of the EIO, PS-sourced beam generation was further advanced with an integrated post-acceleration section and a PS sheet-beam.
\end{abstract}

Keywords-millimetre wave; EIO; pseudospark; electron beam

\section{INTRODUCTION}

Millimetre wave radiation sources in the frequency range of 0.1-10 THz have received increasing interest in recent years because of the demand in the fields of plasma diagnosis, material science, radiotherapy, spectroscopy and advanced communications. Up to date, vacuum electronic technology still remains as the main method to achieve millimetre wave radiation of high power of up to the kilowatt level. Since high quality intense electron beams have been crucial in vacuum electronic devices, thus the PS discharge has attracted a lot of interest recently as a promising source of high quality; high intensity electron beam pulses [1-11]. The PS-sourced electron beam has the ability of self-focusing due to the unique structure and the formation of an ion channel generated by the beam front. Simulations have shown that the PS-sourced electron beam would propagate within background plasma without any applied guiding magnetic field. This makes it an excellent electron beam source for desirable compact millimetre-wave devices. Among various vacuum electronic devices, the EIO has gained considerable attention as a promising millimetre wave oscillation source due to its high gain per unit length and compact configuration [12-16].

In this paper, we use a PS-sourced electron beam instead of the conventional electron beam produced by a thermionic cathode to drive the EIO to achieve more compact devices.

\section{PS-BEAM GENERATION}

The PS discharge is a self-sustained, low pressure (typically 6.5-65 Pa) gas discharge in a hollow cathode/planar anode configuration that can produce a high quality pulsed electron beam, with the highest combined beam current density $\left(>10^{8} \mathrm{~A} / \mathrm{m}^{2}\right.$ ) and brightness (up to $10^{12} \mathrm{Am}^{-2} \mathrm{rad}^{-2}$ ). In addition to the high beam current density, simple structure for easier fabrication, and low vacuum requirement, the PSsourced electron beam does not need any external focusing magnetic field. The electron beam is confined during its propagation by an ion channel formed in the system resulting from the background gas ionization by the beam front itself.

Recently a single-gap PS-sourced electron beam was also studied using a novel methodology of an integrated post acceleration section to achieve high current density and low energy spread beam [17-18]. Fig.1 shows the PS-beam after post-acceleration and Fig. 2 is the beam image.

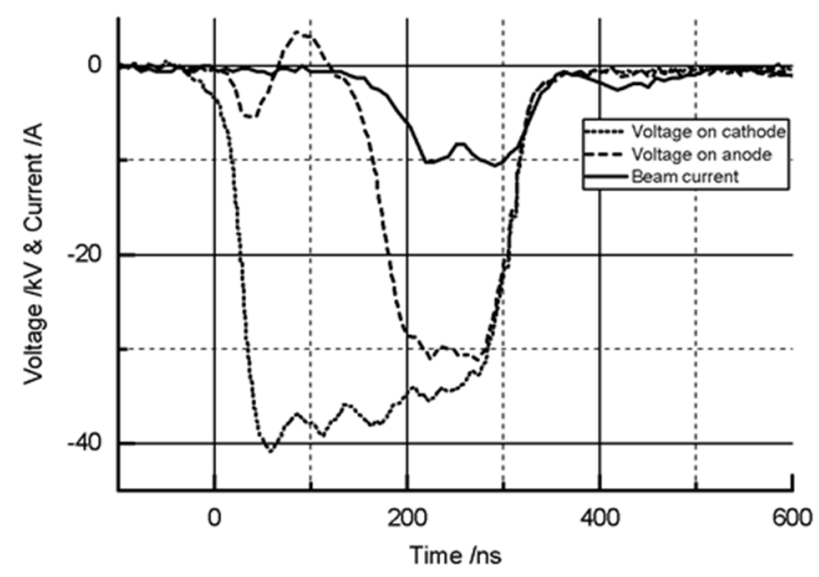

Fig.1 Typical waveforms of post-acceleration of the PS-sourced electron beam, PS discharge

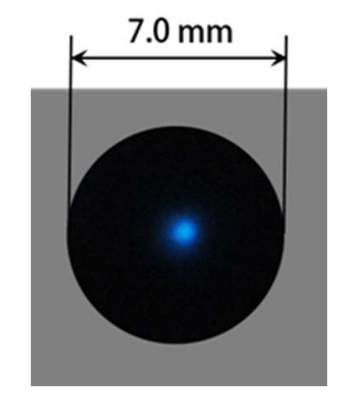

Fig. 2 PS-Beam image captured after post-acceleration 


\section{W-BAND PENCIL BEAM EIO}

A W-band $(75 \mathrm{GHz}-110 \mathrm{GHz})$ EIO circuit operated in the $2 \pi$ mode was selected for the first experiment because of its shorter interaction length, high interaction impedance and high gain per unit length, as compared with a backward wave oscillator. The beam voltage was designed to be centered at $30.5 \mathrm{kV}$, which is suitable for a four-gap pseudospark discharge. This newly developed device combines the merit of a short interaction circuit in the EIO and the high current density property of the pseudospark-sourced electron beam to generate $\mathrm{W}$-band coherent radiation. Experimental results show that with a $35 \mathrm{kV}$ discharge voltage, the EIO successfully produced W-band radiation pulses with $38 \mathrm{~W}$ peak power and $20 \mathrm{~ns}$ duration, which agrees well with the $3 \mathrm{D}$ Particle-in-Cell (PIC) simulations using MAGIC.
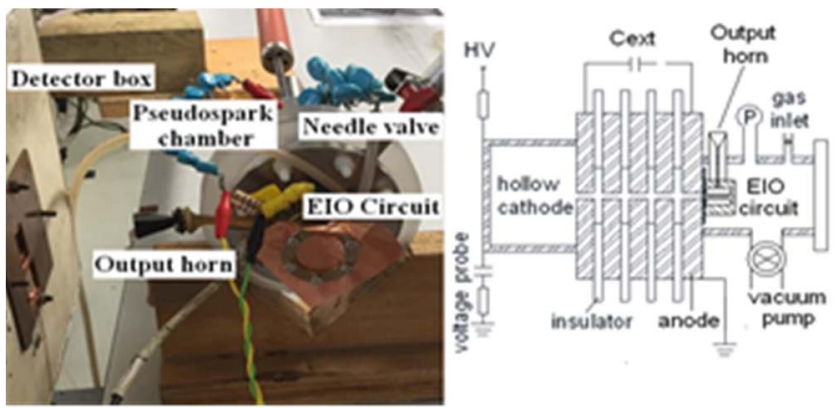

Fig. 3 Experimental setup of the W-band EIO based on a PS-sourced electron beam and the schematic drawing of the experiment.
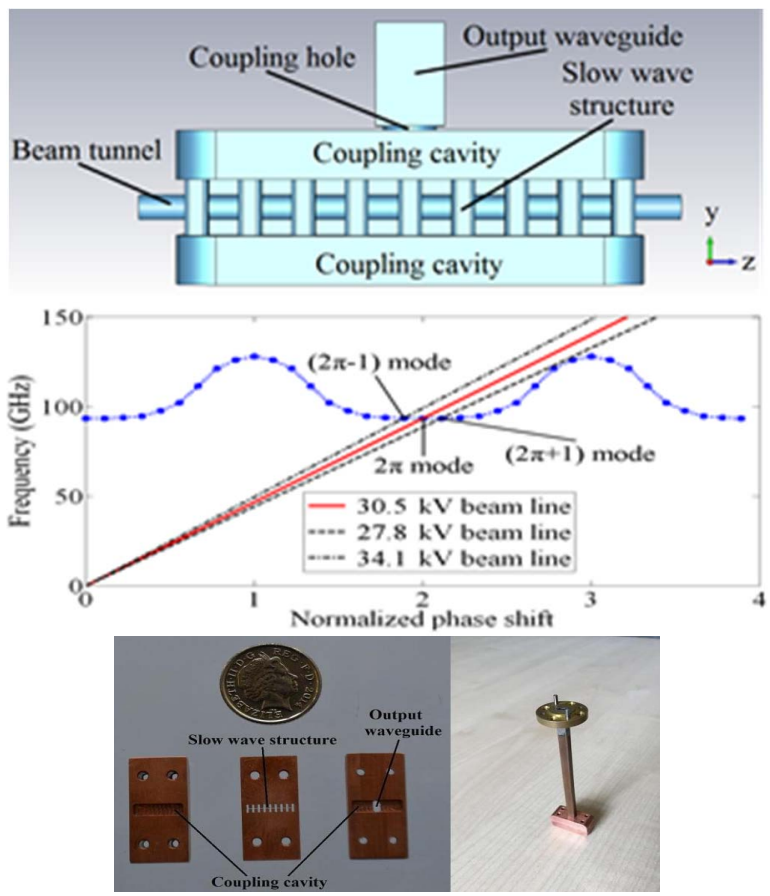

Fig. 4 The layout (top), dispersion (middle) and pictures (bottom) of the EIO

A photograph of the W-band oscillator based on a pseudospark-sourced electron beam and the EIO circuit which includes a schematic drawing of the experiment are shown in Fig. 3. It does not need an external focusing magnetic field and is capable of operating at high repetition rate (up to a few
$\mathrm{kHz}$ ). Fig. 4 shows the layout, dispersion and pictures of the EIO circuit, from which the EIO structure and operating frequency can be seen.

Experimental measurements of the W-band EIO based on the PS-sourced electron beam were carried out at the University of Strathclyde. A four-gap PS discharge chamber which can hold-off a discharge voltage up to $40 \mathrm{kV}$ was used. The discharge voltage swept from $38 \mathrm{kV}$ to $25 \mathrm{kV}$ to achieve the maximum output power. Two W-band horns, one for the output from the EIO and the second horn for the microwave detector, were used. The two horns were separated by a distance of $20 \mathrm{~cm}$. The microwave radiation pulse was measured by a W-band crystal detector. A typical measurement of the voltage, beam current and the microwave signal are shown in Fig. 5. The duration of the microwave waveform agrees well with the simulation.

To estimate the radiation power, the crystal detector was calibrated using a $1.5 \mathrm{~W}, 90-97 \mathrm{GHz}$ solid-state source. The response of the detector at different output powers was recorded. From the calibration data, the radiation power was obtained from the amplitude of the crystal detector output. The peak power of the radiation was found to be about $\sim 38 \mathrm{~W}$. The output frequency was examined using a high pass waveguide cutoff filter which operated above $92 \mathrm{GHz}$. The radiation frequency was found to be above $92 \mathrm{GHz}$ which also coincides with the VNA millimeter wave measurement result of $93.8 \mathrm{GHz}$. A literature review indicates this is the first experiment of an EIO based on a pseudospark-sourced electron beam. It provides a compact and affordable way to generate tens of watts in W-band. Future work will explore a W-band sheet-beam EIO for further output power enhancement.
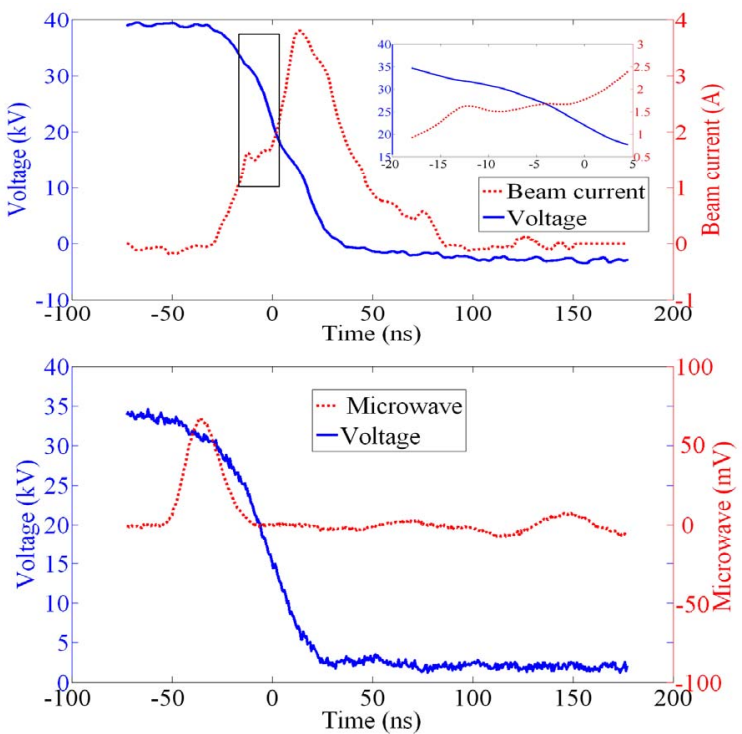

Fig.5 Typical waveforms of the electron beam current, voltage and the microwave pulse.

\section{SHEET BEAM EIO}

In order to improve the output power capacity, it was proposed to combine the advantages of large beam cross 
section in a sheet electron beam and high beam current density in a PS electron beam, aiming to achieve a compact millimetre EIO with high output power. To verify the idea, a G-band PSsourced sheet electron beam EIO was designed and studied. According to the initial analysis in the PS-sourced pencil electron beam $\mathrm{W}$-band EIO in the previous section, the low efficiency is mainly caused by beam current loss in the circuit and large beam velocity spread. Improvements are made in the design to alleviate the disadvantageous effects from these two factors. Additionally, an easier-to-manufacture structure has also been pursued in the design to reduce the fabrication difficulty.

As shown in Fig. 6, the ladder-based slow wave structure is used in the design, which consists of a beam tunnel, eleven gaps, and two coupling cavities. When a short circuit plane is placed at the front end and the back end of the structure respectively, a resonant SWS (RSWS) is formed. The generated electromagnetic (EM) wave in the RSWS is extracted through an output structure.. The structure was made to achieve a relatively easy-to-fabricate configuration, at the cost of tube performance. The beam tunnel directly connects with the coupling cavities at its two sides, without slot waveguides or dumbbell shaped waveguides between the beam tunnel and coupling cavities. The RSWS connects to the standard output waveguide (SOWG) through one coupling waveguide $(\mathrm{CWG})$, rather than two symmetrical $\mathrm{CWG}$ at its two sides. Fig. 7 shows the dispersion curve of the G-band EIO structure. Fig. 8 shows the output power versus sweeping PS discharge voltage and the oscillation start up time Ts. Fig.9 shows some simulation results considering the features of PSsourced electron beam, such as its sweeping voltage-related energy spread, beam current sweep, and EIO structural loss.

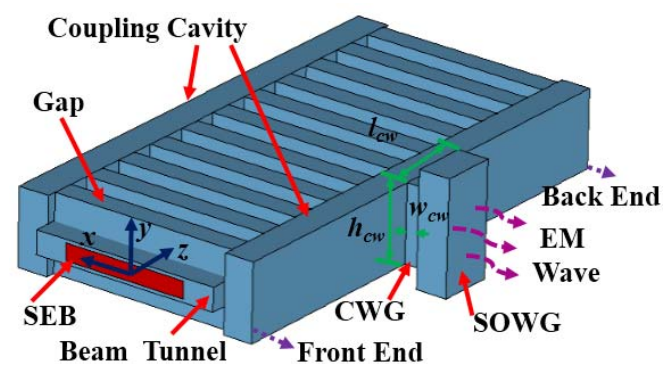

Fig.6 Interaction circuit composed of a RSWS and an output structure

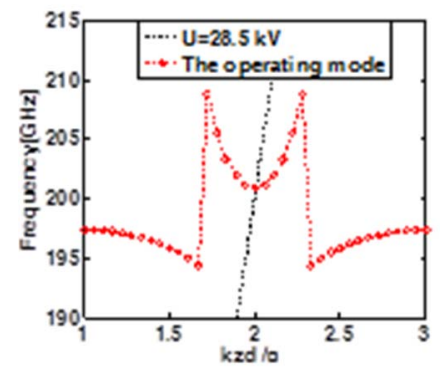

Fig.7 The dispersion curve of the G-band sheet beam EIO

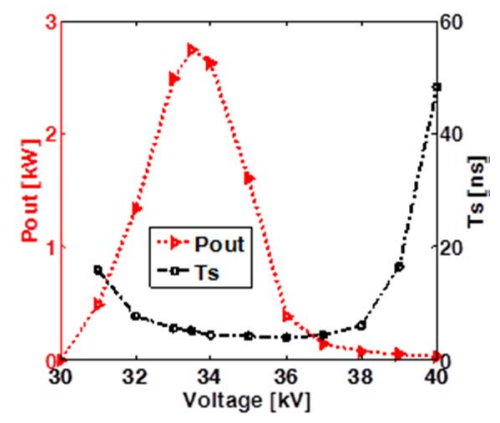

Fig.8 G-band PS sheet beam EIO output power prediction against PS discharge voltage and the oscillation start up time Ts

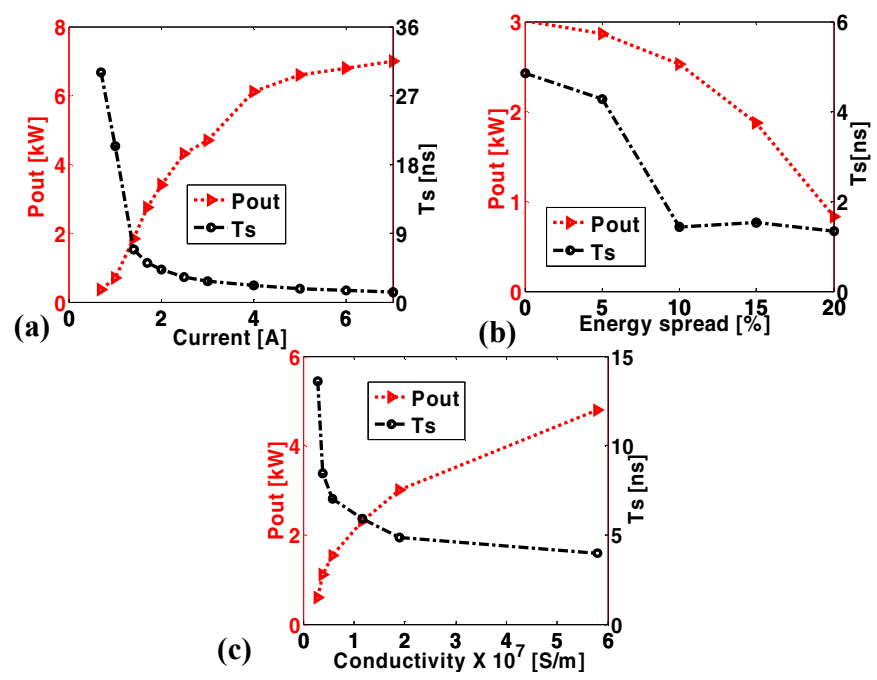

Fig.9 Simulation results considering some actual situations. Pout and Ts versus (a) beam current (b) energy spread and (c) conductivity.

From the simulation results, it can be seen that the sheetbeam G-band EIO will have a much greater output power.

\section{CONCLUSIONS}

The investigation of millimeter wave EIO have been carried out recently with the first experiment from a W-band pencil beam EIO using a 4-gap PS-sourced electron beam and an output power of $38 \mathrm{~W}$ was achieved. A G-band sheet beam EIO was further studied with about $2 \mathrm{~kW}$ output power predicted. The PS-sourced electron beam is also studied with an integrated post acceleration section to improve the beam energy spread, which will enhance greatly the output power of millimeter wave devices.

\section{REFERENCES}

[1] J. Christiansen and C. Schultheiss, "Production of high-current particle beams by low-pressure spark discharges", Z. Physik A 290, 35-41, 1979

[2] D. Bowes, H. Yin, W. He, A.W. Cross, K. Ronald, A.D.R. Phelps, D. Chen, P. Zhang, X. Chen and D. Li, "Visualization of a PseudosparkSourced Electron Beam", IEEE Transaction on Plasma Science, 42, 10, pp2826-2827, 2014

[3] D. Bowes, H. Yin, W. He, L. Zhang, A.W. Cross, K. Ronald, et al. "Xray emission as a diagnostic from pseudospark-sourced electron beams", Nuclear Instruments \& Methods in Physics Research Section B, V. 335. No. P. 74-77, 2014 
[4] J. Zhao, H. Yin, L. Zhang, G. Shu, W. He, J. Zhang, Q. Zhang, A. D. R. Phelps, and A. W. Cross, "Influence of the electrode gap separation on the pseudospark-sourced electron beam generation," Phys. Plasmas, vol. 23, 073116, July 2016

[5] H. Yin, A. W. Cross, A. D. R. Phelps, D. Zhu, W. He, and K. Ronald, "Propagation and post-acceleration of a pseudospark-sourced electron beam", J. Appl. Phys., vol. 91, pp. 5419-5422, 2002

[6] J. Zhao, H. Yin, L. Zhang, G. Shu, W. He, A. D. R. Phelps, A. W. Cross, L. Pang, and Q. Zhang, "Study of the beam profile and position instability of a post-accelerated pseudospark-sourced electron beam", Physics of Plasmas vol. 24, 033118, 2017

[7] H. Yin, G. R. M. Robb, W. He, A. D. R. Phelps, A. W. Cross, and K. Ronald, "Pseudospark-based electron beam and Cherenkov maser experiments", Physics of Plasmas, vol. 7, pp. 5195-5205, 2000

[8] H. Yin, W. He, A.W. Cross, A.D.R. Phelps, and K. Ronald, "Singlegap pseudospark discharge experiments", J. Appl. Phys. V. 90. No. 7. P. 3212-3218, 2001

[9] H. Yin, A.W. Cross, W. He, A.D.R Phelps, and K. Ronald, "Pseudospark experiments: Cherenkov interaction and electron beam post-acceleration”, IEEE Trans. Plasma Sci. V. 32. No. 1. P. 233-239, 2004

[10] H. Yin, A.W Cross, W. He, A.D.R Phelps, K. Ronald, D. Bowes, and C.W. Robertson, "Millimeter wave generation from a pseudosparksourced electron beam", Phys. of Plasmas. V. 16. No. 6. P. 063105 , 2009

[11] W. He, L. Zhang, D. Bowes, H. Yin, K. Ronald, A. D. R. Phelps, and A. W. Cross, "Generation of broadband terahertz radiation using a backward wave oscillator and pseudospark-sourced electron beam," Appl. Phys. Lett., vol. 107, no. 13, 133501, Sept. 2015
[12] Y. Yin, W. He, L. Zhang, et al.,"Preliminary design and optimization of a G-band extended interaction oscillator based on a pseudosparksourced electron beam," Phys. Plasmas, vol. 22, no. 7, pp. 073102-1073102-6, Jul. 2015

[13] Y. Yin, W. He, L. Zhang, H. Yin, C. W. Robertson and A. W. Cross, "Simulation and Experiments of a W-band Extended Interaction Oscillator based on a pseudospark-sourced electron beam," IEEE Trans. Electron Devices, vol. 63, no. 1, pp. 512 - 516, Jan. 2016

[14] G. Shu, W. He, L. Zhang, Y. Yin, J. P. Zhao, A. W. Cross, A. Phelps, "Study of a $0.2 \mathrm{THz}$ extended interaction oscillator driven by a pseudospark-sourced sheet electron beam," IEEE Trans. Electron Devices, vol. 63, no. 12, pp.4955-4960, Dec. 2016

[15] R. Dobbs, B. Steer, "Extended interaction sources above 220," 36th Int. Conf. Infrared, Millimeter and Terahertz Waves (IRMMW-THz), Houston, USA, pp. 1, 2011

[16] J. Pasour, E. Wright, K. Nguyen, and B. Levush, "Compact, multi-kW sheet beam oscillator at $94 \mathrm{GHz}, "$ 41th Int. Conf. Plasma Sciences (ICOPS), Washington DC, USA, pp. 1., 2014

[17] J. Zhao, H. Yin, L. Zhang, G. Shu, W. He, Q. Zhang, A. D. R. Phelps, and A. W. Cross, "Advanced post-acceleration methodology for pseudospark-sourced electron beam", Physics of Plasmas 24 (2), 023105, 2017

[18] J. Zhao, H. Yin, L. Zhang, W. He, Q. Zhang, A. D. R. Phelps, and A. W. Cross, "Experiments on W-band extended interaction oscillator with pseudospark sourced post-accelerated electron beam", Physics of plasmas 24060703,2017 